\title{
Predicting liquid loss of frozen and thawed cod from hyperspectral imaging
}

\author{
Kathryn E. Anderssen ${ }^{a, *}$, Svein Kristian Stormo ${ }^{a}$, Torstein Skåra ${ }^{b}$, Martin H. Skjelvareid ${ }^{c}$, \\ Karsten Heia ${ }^{\text {a }}$ \\ a Department of Seafood Industry, Nofima AS, P.O. Box 6122, 9291, Troms $\phi$, Norway \\ ${ }^{\mathrm{b}}$ Department of Processing Technology, Nofima AS, P.O. Box 8034, 4068, Stavanger, Norway \\ ${ }^{\mathrm{c}}$ Department of Computer Science and Computational Engineering, UiT the Arctic University of Norway, PO Box 143, 8049, Bod $\phi$, Norway
}

\section{A R T I C L E I N F O}

\section{Keywords:}

Hyperspectral imaging

Fish

Cod

Storage

Freezing

Classification

\begin{abstract}
A B S T R A C T
As the ability to appraise the quality of every fish in a delivery in a consistent, objective, and rapid manner has numerous advantages for both sellers and buyers, there has been much research into methods to achieve this. One possible proxy for quality assessment is liquid loss, which correlates with undesirable sensory attributes. This study evaluated whether hyperspectral imaging could predict liquid loss on samples that had undergone a program of freezing and thawing. Vacuum-packaged cod loins were split into two groups, one which was kept chilled and the other that underwent a program of freezing and thawing. Multivariate analysis of the hyperspectral imaging data on the chilled samples could predict liquid loss with good accuracy for samples that underwent no further processing. Analysis of data from the hyperspectral images of the frozen and thawed cod samples also showed a good ability to predict their liquid loss. These results indicate that hyperspectral imaging is a promising method for non-invasive quality monitoring of cod products in different processing states. Also, whereas previous research had been unable to predict sample processing protocols, improvements to the hyperspectral imaging technology now enables identification of samples based on freezing and thawing procedures.
\end{abstract}

\section{Introduction}

There has been an increased focus on the quality of seafood products in recent years. Improving product quality is a way to increase profitability while maintaining sustainability, enabling more revenue to be generated from a catch without increasing its size. Furthermore, consumers are beginning to demand higher quality products, such that high product quality is no longer simply a factor of price, but sometimes necessary to obtain market entry at all (Altintzoglou \& Heide, 2016; Peavy, Work, Riley, 1994). Therefore, the ability to rapidly sort raw material by quality would greatly benefit the processors. Initial quality control on raw materials enables a more even production quality and reduces the risk in of a product later being downgraded. Removing lower quality raw materials from a product line has also been shown to improve margins and lower labor costs (Zugarramurdi, Parin, Gadaleta, Carrizo, Lupin, 2004).

Despite its importance, the property of "quality" is difficult to both define and quantify. It is frequently applied subjectively and what is considered a "good" quality product may vary from market to market
(Cardello, 1995). A variety of different approaches have been used to attempt to provide objective, reproducible evaluation of sample quality. The quality index method (QIM) is one of the most common for determining freshness (or remaining shelf life) of chilled (non-frozen) fish (Bonilla, Sveinsdottir, Martinsdottir, 2007; Warm, Bøknæs, Nielsen, 1998). However, the technique is labor intensive, such that its use is limited to spot checks. Also, even with trained personnel, the method is subjective and can vary between evaluators, or even from day to day with the same evaluator.

A promising method for quality control in the seafood industry is hyperspectral imaging (Cheng \& Sun, 2014; Siche et al., 2016; Sun, 2010). Whereas a regular camera has three channels-red, green and blue - a hyperspectral camera measures numerous wavelengths. By observing which wavelengths are absorbed by the fish tissue, a characteristic spectral fingerprint can be taken for a sample and used to understand its composition and structure. Because it is rapid and non-invasive, it can be performed easily in an industrial setting, for example to monitor products on a conveyor belt. The method can also be used on products in both the chilled and frozen state. To date,

\footnotetext{
* Corresponding author.

E-mail address: kate.anderssen@nofima.no (K.E. Anderssen).
} 
hyperspectral imaging has been used to perform a variety of quality control measurements on fish products (Cheng, Niccolai, Sun, 2017), such as blood content (Skjelvareid, Heia, Olsen, Stormo, 2017), fillet composition (ElMasry \& Wold, 2008), and fillet freshness (Sivertsen, Kimiya, Heia, 2011). It can also aid in the evaluation of frozen and thawed fish products. Previous research has found that hyperspectral imaging could distinguish between fresh and thawed products (Cheng, Sun, Pu, 2016; Zhu, Zhang, He, Liu, Sun, 2013), identify freezer burn in salmon (Xu \& Sun, 2017), and determine freezing temperature of frozen cod fillets (Washburn, Stormo, Skjelvareid, Heia, 2017).

One objective measure that can be applied to estimate sample quality is liquid loss. Liquid loss is an indication of post mortem changes (Ofstad et al., 1996) and cellular damage to the flesh. It is associated with a variety of undesirable sensory properties such as dryness, toughness and chewiness after cooking (Hurling \& McArthur, 1996; MacCallum, Laishley, Dyer, Idler, 1966). Raw fish that have experienced high liquid loss tend to have a softer texture. This makes them both less desirable from a sensory perspective and more difficult to work with in processing machines, as the flesh tends to tear more easily. Previous research investigated whether hyperspectral imaging could be used to predict liquid loss of salmon (He, Wu, Sun, 2014). Samples were measured via hyperspectral imaging, stored for a period of time and the liquid loss measured. Sample liquid loss could be predicted with good accuracy from the hyperspectral data. Other researchers used hyperspectral imaging to predict drip loss of thawed grasscarp with storage time (Cheng et al., 2016). However, the liquid loss is not only dependent on intrinsic properties. Subsequent handling will also influence the amount of liquid loss the tissue experiences. Common processing methods such as freezing and thawing have been shown to increase the amount of liquid loss (Einen, Guerin, Fjæra, Skjervold, 2002; Fagan, Gormley, Mhuircheartaigh, 2003; Mørkøre \& Lilleholt, 2007). In particular, a slow freezing-rate will produce more damage to the tissue. This enables larger ice crystals to form in the tissue during the freezing process, leading to more cellular damage (Dalvi-Isfahan et al., 2019; Petzold \& Aguilera, 2009). This study set out to test whether hyperspectral imaging could be used to predict liquid loss from vacuum packaged cod fillet portions at different stages of processing. Measurements were performed on samples in the chilled, frozen and thawed states.

\section{Materials and methods}

\subsection{Hyperspectral imaging equipment}

The hyperspectral imaging setup used in this study is shown in Fig. 1. It operates in the interactance mode, which has the advantage of

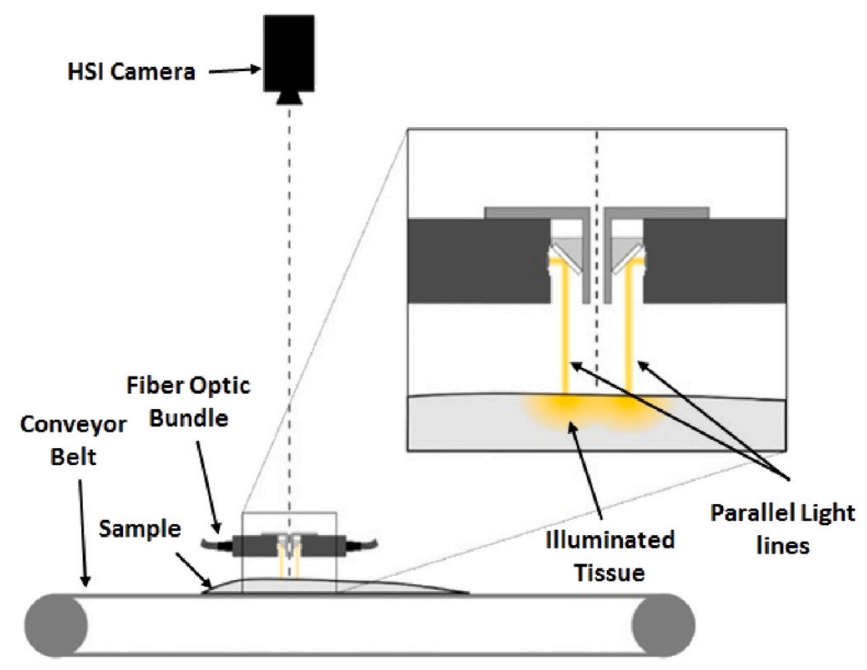

Fig. 1. Schematic of the hyperspectral interactance imaging setup. measuring farther below the sample surface than the more commonly used diffuse reflectance mode. Two light lines are used, as previous work has shown that dual illumination improves signal to noise compared to a single source (Sivertsen et al. 2009; Wold et al., 2006). Dual illumination also avoids the problem of being unable to obtain a signal at sample edges. The camera line is placed between the two light lines. The illumination source was a pair of custom built fiber optics (Fiberoptics Technology Inc. Connecticut, USA). These were fitted with custom made collimating lenses (Optec S.P.A., Milano, Italy). Each light line contains six fiber optic bundles with each fiber optic fed by a $150 \mathrm{~W}$ halogen lamp focused with an aluminium reflector (International Light Technologies, Massachusetts, USA, model L1090). The light lines are each approximately $400 \mathrm{~mm}$ long and $5 \mathrm{~mm}$ in width, and together have a total of $1800 \mathrm{~W}$ of electrical input power.

The hyperspectral camera used for this study was the HySpex VNIR1024 (Norsk Elektro Optikk, Oslo, Norway). The hyperspectral camera field of view is $0.56 \mathrm{~mm} \times 300 \mathrm{~mm}$ with a pixel size of $0.28 \mathrm{~mm} \times 0.56$ $\mathrm{mm}$. Light is measured in the $430-1000 \mathrm{~nm}$ spectral region with approximately a $5.4 \mathrm{~nm}$ resolution. Samples were imaged on a conveyor belt traveling at $40 \mathrm{~cm} / \mathrm{s}$, a rate that conforms to the industrial production capacity limit of approximately one fillet per second. The hyperspectral camera operates in pushbroom mode, scanning the sample line by line at 400 frames per second as it moves through the field of view. The successive frames $F(\lambda, x)$ are stored as a hyperspectral image $R_{i}(\lambda, x, y)$. In order to correct for changes in camera sensitivity and illumination with time, a calibration sample of Teflon, which has stable optical properties (Tsai, Allen, Hanssen, Wilthan, Zeng, 2008), is measured before each set. The calibration sample used is a square 300 $\mathrm{mm}$ on each side with a thickness of $25 \mathrm{~mm}$. One hundred successive frames of the Teflon target are averaged together to calculate a reference frame $R_{a}(\lambda, x)$. The absorption of the interactance images are then calibrated as $I(\lambda, x, y)=-\ln \left(R_{i}(\lambda, x, y) / R_{a}(\lambda, x)\right)$, where $R_{i}(\lambda, x, y)$ is the hyperspectral image of the packaged fillet section.

\subsection{Fish samples}

Atlantic cod caught by jig on fishing grounds close to Troms $\varnothing$, Norway were killed by a blow to the head, immediately gutted and bled for $30 \mathrm{~min}$. The fish were transported to Nofima (whole and gutted) and kept on ice for two days prior to filleting. After filleting, the loins of each fish were cut into uniform pieces $(\mathrm{n}=73,112.3 \pm 5.6 \mathrm{~g}$ ), vacuumpacked in sous vide plastic pouches, and kept on ice prior to subsequent processing. Vacuum was drawn to $99 \%$ and the sous vide pouches had a $20 \mu \mathrm{m}$ polyamide inside layer and $70 \mu \mathrm{m}$ polyethylene outside layer, giving an $\mathrm{O}_{2}$ permeability of $45 \mathrm{~cm}^{3} /\left(\mathrm{m}^{2} \mathrm{~d}\right.$ bar)).

All samples immediately underwent hyperspectral measurements in the chilled, packaged state. The batch of samples was split into two groups: one group $(\mathrm{n}=33)$ that was kept chilled on ice and one group ( $\mathrm{n}$ $=40$ ) that underwent a program of freezing and thawing before liquid loss measurements. Two freezing and two thawing procedures were used: a fast-freezing procedure, which involved blast freezing the samples at $-40{ }^{\circ} \mathrm{C}$ (air speed $3 \mathrm{~m} / \mathrm{s}$ ) and a slow-freezing procedure, which involved freezing the samples to $-20{ }^{\circ} \mathrm{C}$ in still air. The fast-thawing procedure involved thawing the samples in $4{ }^{\circ} \mathrm{C}$ circulating water for $1 \mathrm{~h}$ while the slow procedure used still air at $4{ }^{\circ} \mathrm{C}$ for $18 \mathrm{~h}$. The frozen storage period was five days. All samples were stored at $-20^{\circ} \mathrm{C}$ for $24 \mathrm{~h}$ prior to spectroscopy measurements in the frozen state to allow them to equilibrate at $-20{ }^{\circ} \mathrm{C}$ to avoid temperature differences affecting the spectra. The last round of spectroscopy and liquid loss measurments were performed immediately after thawing.

\subsection{Liquid loss measurement}

Liquid loss was collected directly from the vacuum packages. The vacuum packed samples containing fish muscle and expelled liquid were opened and the expelled liquid was separated from the solid material by 
filtering using a sieve (mesh size: $1 \mathrm{~mm}$ ).

Liquid loss (LL, \%) was determined according to the formula:

$L L=\frac{\left(m_{0}-m_{L}\right)}{m_{0}} \times 100 \%$

where $m_{0}$ is the initial weight of the loin, and $m_{L}$ is the weight of the loin after packaging or packaging plus a freezing/thawing cycle.

\subsection{Data analysis}

\subsubsection{Image pre-processing}

Processing of hyperspectral images was performed using IDL (Exelis Visual Information Solutions, Bracknell, United Kingdom). Large hyperspectral images of sample batches were split into individual images of each packaged sample, image calibration performed and regions of interest (ROI) were manually selected in the center of the samples. The ROIs were selected to avoid any defects or image distortions and the spectra were exported. Further analysis of the data was performed using the R Programming language (Free Software Foundation Inc., Boston, MA). Principal component analysis was performed on the spectra from each sample to identify pixel outliers, which were removed from the set of spectra, and then the spectra were averaged to produce a single spectrum for each sample. Spectra were normalized to unit area below the spectrum curve, and the first derivative of the normalized spectrum was used for analysis. This serves to emphasize subtle differences in the spectra and acts to remove baseline effects (Burger \& Geladi, 2007). Principal component analysis was used to evaluate the initial hyperspectral data of the samples in the chilled state in order to identify any unexpected patterns or outliers. The samples were uniformly distributed in the PCA space, indicating there were no hidden data trends that could confound later analysis.

\subsubsection{Liquid loss prediction}

Prediction of liquid loss was performed using a partial least squares regression (PLSR) from the R 'pls' package (Mevik, Wehren, Liland, 2019; Mevik \& Wehrens, 2007). For most analyses, the root mean square error of prediction (RMSEP) initially decreases with increased number of components and then plateaus, indicating that additional components do not provide any further information. The number of components used for prediction was selected at the start of the plateau in the RMSEP curve.

\subsubsection{Wave length optimization}

In addition to analysis with the full spectra, wavelength optimization was performed using a genetic algorithm (Bangalore, Shaffer, Small, 1996). Wavelength optimization has several advantages. Spectral measurements frequently contain wavelength regions that are irrelevant to the property under study. When these sections are included in analysis, developed models must compensate for variance in these regions even though they do not contribute information. Limiting wavelengths to spectral regions that contain information frequently improves prediction results and decreases the number of components necessary in the model. However, wavelength optimization is prone to overfitting and care must be taken to avoid producing unrealistically optimistic models (Anderssen, Dyrstad, Westad, Martens, 2006). A double cross-validated approach was used in this study. For optimization, the data set was randomly divided into a training and a test set. The genetic algorithm begins with a population of initially randomly selected wavelengths, commonly referred to as "chromosomes". PLS models using the samples in the training set are created using the subsets of wavelengths in these chromosomes and then the performance of each of the models is evaluated using the test set. The best performing chromosomes are mated together to produce a new population of chromosomes. This new population of chromosomes is a random combination of the wavelengths in the parent chromosomes, with some chance of random mutation. The data set is randomly divided into a new training set and test set and the performance of the new generation of chromosomes are tested. By changing the samples present in the training and test sets between each generation, the likelihood of overfitting is greatly decreased. The best performing chromosomes are again mated together to produce a new set of chromosomes and the process is repeated for a given number of generations. The wavelengths in the best performing chromosome are then returned. The optimization was performed using the "genalg" genetic algorithm package in $\mathrm{R}$ with a population size of 200 and was repeated for 500 generations.

\subsubsection{Data classification}

Data classification in PCA space was performed using a KNN (knearest neighbors) algorithm using $\mathrm{k}=3$ (Cover \& Hart, 1967). Here, the closest samples to a new sample are used to classify new data. Cross validation was performed using the "Leave One Out" method and the predicted values were compared to actual values to evaluate performance.

\section{Results}

\subsection{Effect of freezing and thawing protocol on liquid loss}

The results show the significant impact freezing and thawing protocol can have on product quality, shown Fig. 2. Statistical significance was evaluated using Welch's $t$-test (Welch, 1947). For samples kept in the chilled state, mean liquid loss was $1.3 \%$ while for samples that were frozen and thawed, mean liquid loss jumped to $4.2 \%(\mathrm{t}=9.3, \mathrm{p}=$ 6.7e-13). However, the amount of liquid loss was heavily impacted by the processing protocol. Liquid loss for quickly frozen samples had a mean value of $3.0 \%$ while samples that were frozen slowly had nearly double the liquid loss, with a mean of $5.2 \%(t=4.4, p=9.8 \mathrm{e}-5)$. Although thawing protocol appeared to have some influence on results, the effect did not appear to be significant $(t=0.63, p=0.53)$. Mean liquid loss for quickly thawed samples was $3.9 \%$ while $4.2 \%$ for slowly thawed samples. When results were broken down further by both freezing and thawing protocols, there was little difference in liquid loss between the fast frozen samples that had been thawed quickly and those that had been thawed slowly $(t=0.6 \mathrm{p}=0.49)$. Similarly for the slow frozen samples, there was virtually no difference between the amount of liquid loss for the fast and slow thawed samples $(t=0.4 \mathrm{p}=0.69)$.

\subsection{Prediction of liquid loss from measurements in the chilled state}

Data from the initial hyperspectral images of all the samples in the chilled state was analyzed to predict liquid loss. For the chilled samples with no subsequent processing, it was possible to predict the liquid loss from the hyperspectral images with good accuracy, as shown in Fig. 3A. RMSEP for that sample set was $0.32 \%$ with an $\mathrm{R}^{2}$ of 0.78 using nine components. This replicates in white fish the findings of the previous study (He et al., 2014), which demonstrated that liquid loss in salmon could be predicted from hyperspectral images of the samples in the chilled state. The number of components used and accuracy of prediction is similar between the salmon study and the results here. On the other hand, attempts to predict the liquid loss of the processed samples using their initial hyperspectral images taken in the chilled state (before the samples had experienced freezing and thawing) produced, as expected, much worse results. The average error of prediction was $1.16 \%$ with an $\mathrm{R}^{2}$ of 0.46 . Only two components were used, but RMSEP increased with additional components. While there still appears to be some relationship between liquid loss and the initial properties of the fish samples that underwent freezing and thawing, previous research that shows that subsequent processing of the samples is the dominant factor for liquid loss (Einen et al., 2002; Fagan et al., 2003; Mørkøre et al., 2007), which is reflected in the results shown in Fig. 3B. Therefore, attempting to estimate liquid loss of processed products from the 


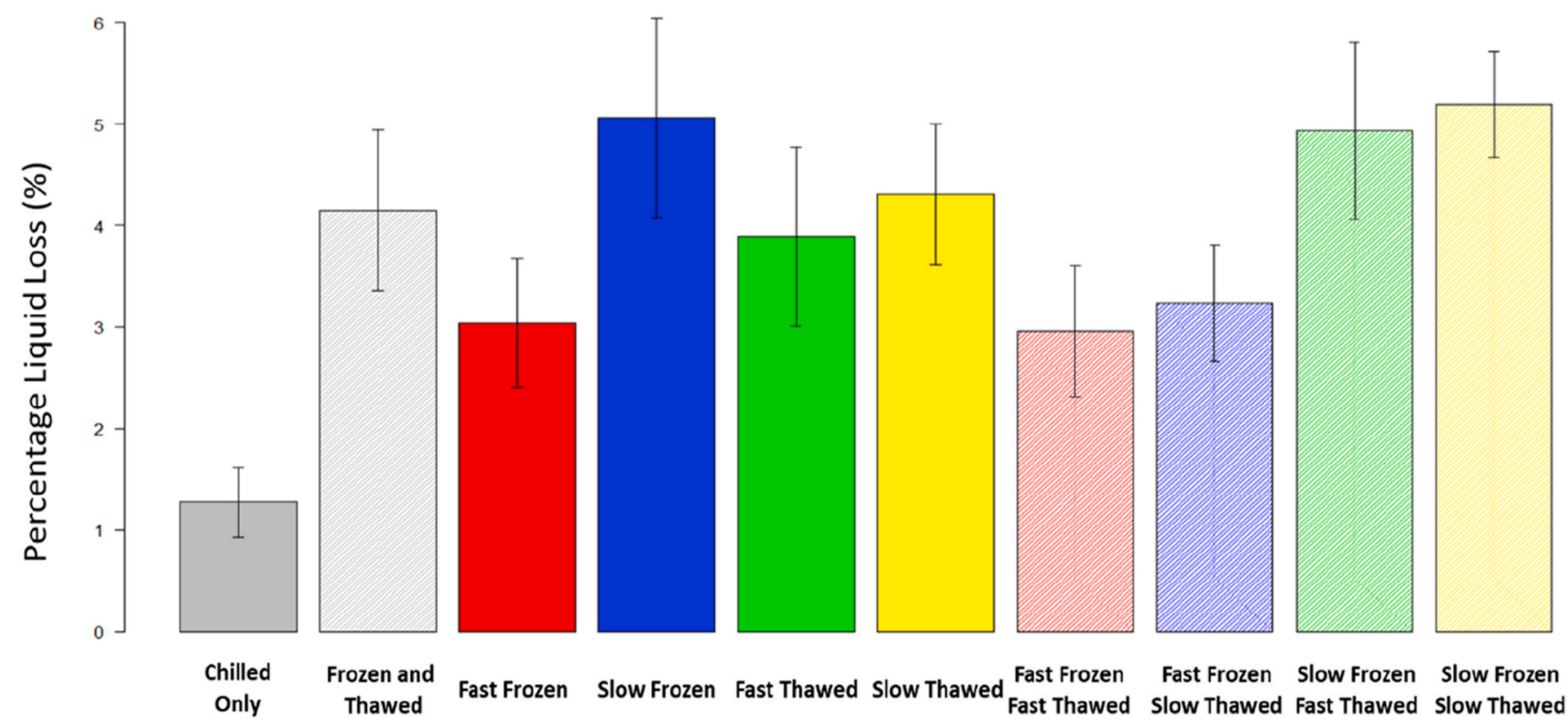

Fig. 2. Mean sample liquid loss with standard deviation for the different freezing and thawing protocols.

A)

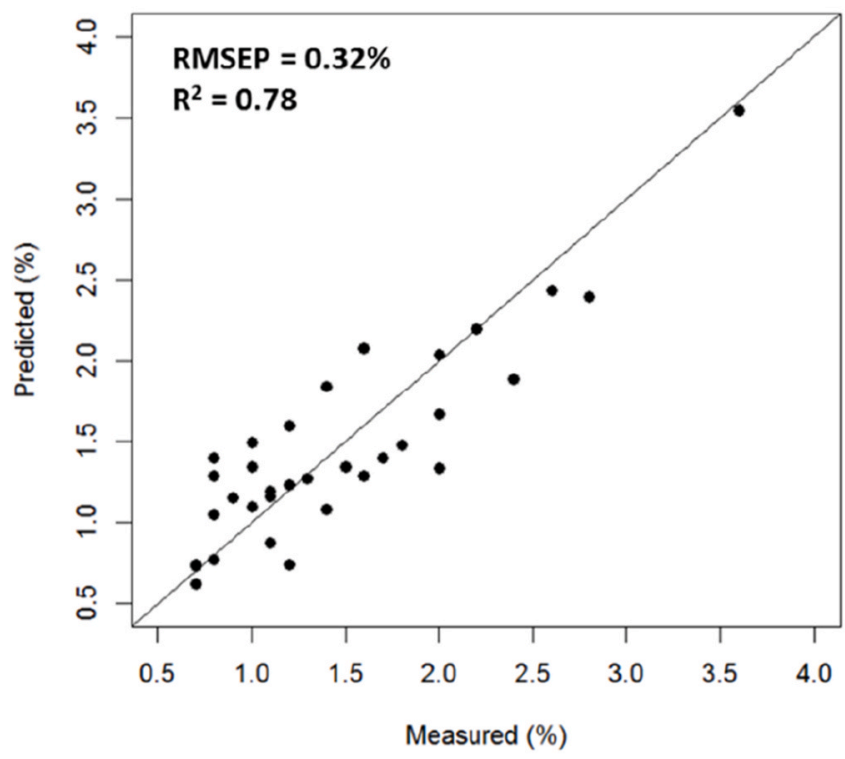

B) Subsequent Freezing and Thawing

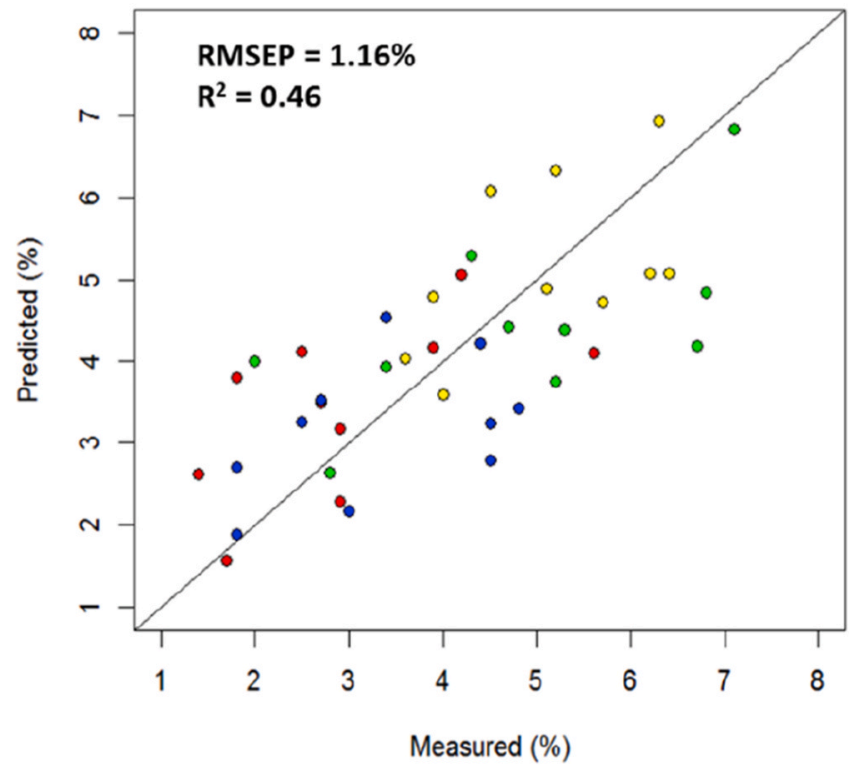

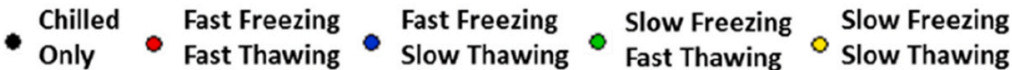

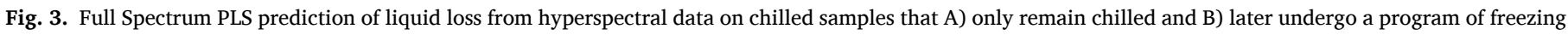
and thawing.

initial raw material before processing will only produce marginal results.

After wavelength optimization, for the first set of samples (Fig. 4A), RMSEP was moderately improved to $0.27 \%\left(\mathrm{R}^{2}=0.85\right)$ but only required four components for prediction. Little improvement was seen on the second set of samples (Fig. 4B), with wavelength optimization. RMSEP was improved to $1.0 \%$ and used 3 components. This provides confidence that the chosen method of wavelength optimization is not overfitting and leading to spurious data correlations.

\subsection{Prediction of liquid loss from measurements in the frozen state}

As found in a previous study (Washburn et al., 2017), it was possible using a PCA to separate hyperspectral images of frozen state samples by freezing protocol, Fig. 5. Sample freezing protocol could be identified with $100 \%$ accuracy by KNN. Prediction of liquid loss from hyperspectral data of the processed samples in the frozen state was significantly improved compared to the results from them in the chilled state (prior to freezing), Fig. 6. Using ten components, RMSEP was $0.69 \%$ 
A)

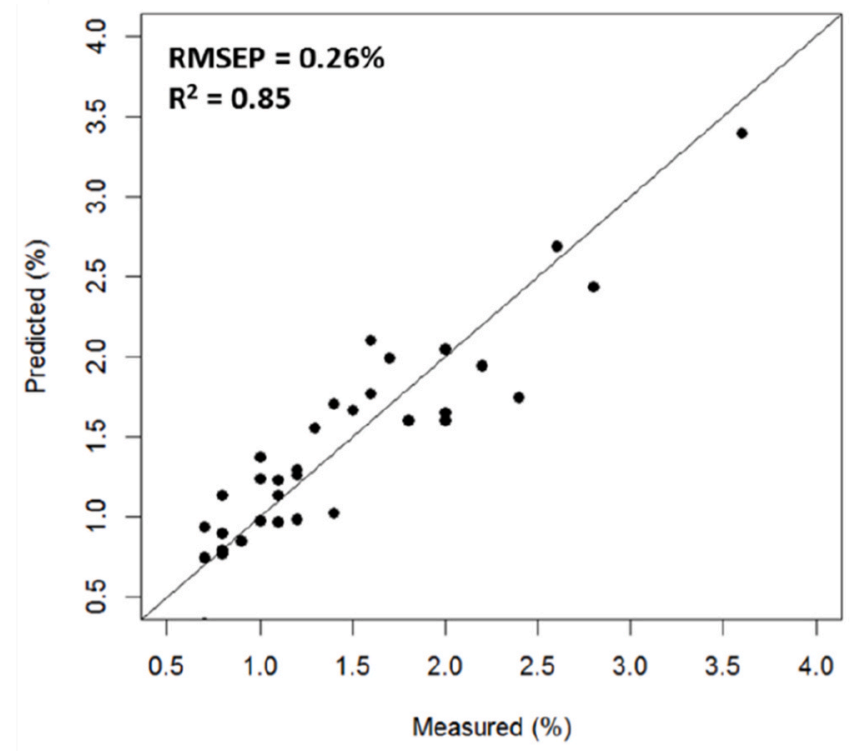

B) Subsequent Freezing and Thawing

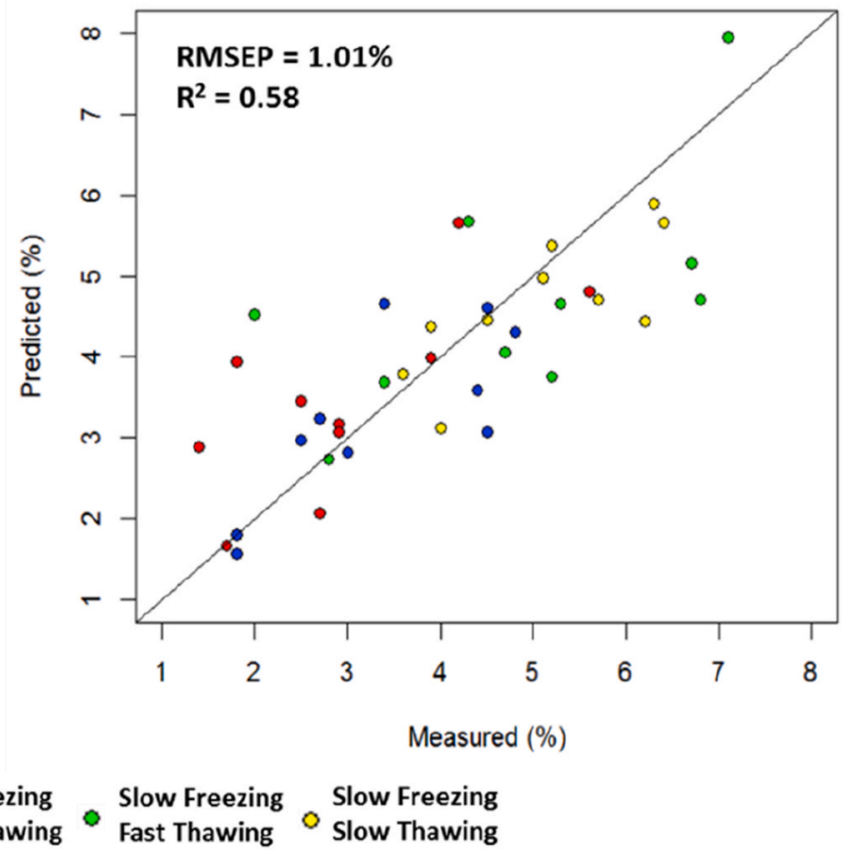

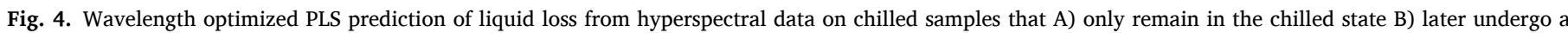
program of freezing and thawing.

with an $\mathrm{R}^{2}$ of 0.81 . After wavelength optimization, only four components were required to achieve a RMSEP of $0.58 \%$ with an $\mathrm{R}^{2}$ of 0.87 .

\subsection{Prediction of liquid loss from thawed state measurements}

Finally, liquid loss predictions were performed based on hyperspectral images of the processed samples in the thawed state. Results are shown in Fig. 7. Prediction using the complete spectrum had an RMSEP of $0.41 \%\left(R^{2}=0.94\right)$ using nine components. For the wavelength optimized, RMSEP was similar but only required five components.

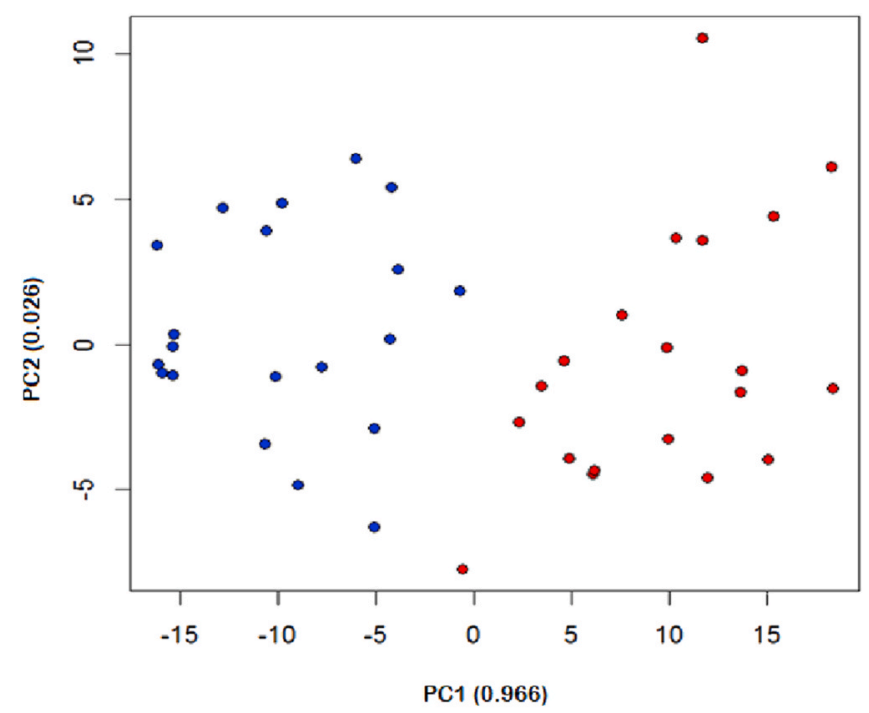

Fig. 5. PCA of hyperspectral data on fillets in the frozen state for samples frozen at $-20{ }^{\circ} \mathrm{C}$ (red) and $-40{ }^{\circ} \mathrm{C}$ (blue). (For interpretation of the references to colour in this figure legend, the reader is referred to the Web version of this article.)

\subsection{Prediction of liquid loss for combined fresh and thawed measurements}

In some situations, there may be a mixture of fish that are in the fresh and thawed state that needs quality evaluation. For example, a delivery to a producer may consist of catches from several different vessels, each with its own handling protocol. To evaluate how well the method worked for predicting liquid loss on a combination of chilled and thawed samples, analysis was performed on the combined set of chilled and processed samples. While the technique generally performed well (RMSEP $0.77 \%, \mathrm{R}^{2}=0.82$ seven components for the full spectrum, RMSEP $0.62 \%, \mathrm{R}^{2}=0.88$ five components for the wavelength optimized), performance was degraded compared to both the liquid loss predictions on the fresh or thawed samples individually, shown Fig. 8.

However, examination of the principal component of the data, Fig. 9, shows a clear delineation between chilled and thawed samples; KNN classification identified sample state with $100 \%$ accuracy. Therefore, a more practical approach would be to initially classify fillets as fresh or thawed, and then to use models optimized for a particular sample state to produce better results.

\subsection{Differentiation of freezing and thawing protocols in the thawed state}

Previous research attempted to identify the freezing and thawing protocols of thawed fillets using hyperspectral imaging (Washburn et al., 2017). The classification attempts had a success rate similar to random chance. With the improvements made to the imaging system (e.g. stronger illumination, closer light lines to improve signal to noise) since the previous study, classification of the thawed fillets by freezing and thawing protocol was attempted again.

With the updated equipment, the results were much better. Based on hyperspectral images data of the thawed samples, freezing protocol could be correctly identified with $95 \%$ accuracy, thawing protocol with 93\% accuracy, and both the freezing and thawing protocol with $93 \%$ accuracy. All samples that had both fast-freezing and fast-thawing or slow-freezing and slow-thawing were correctly identified. More 
A)

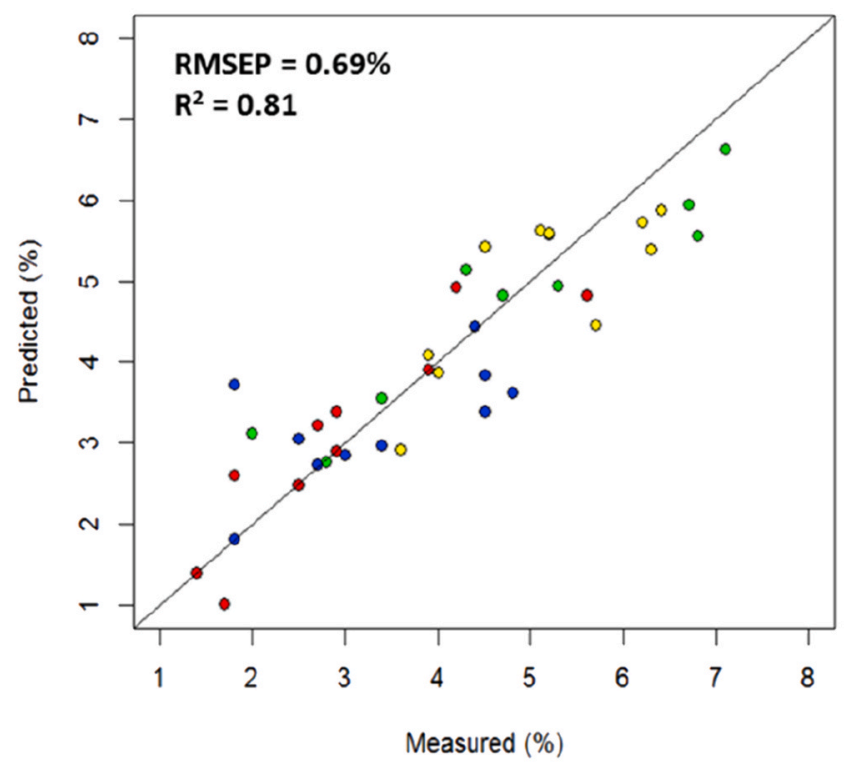

B)

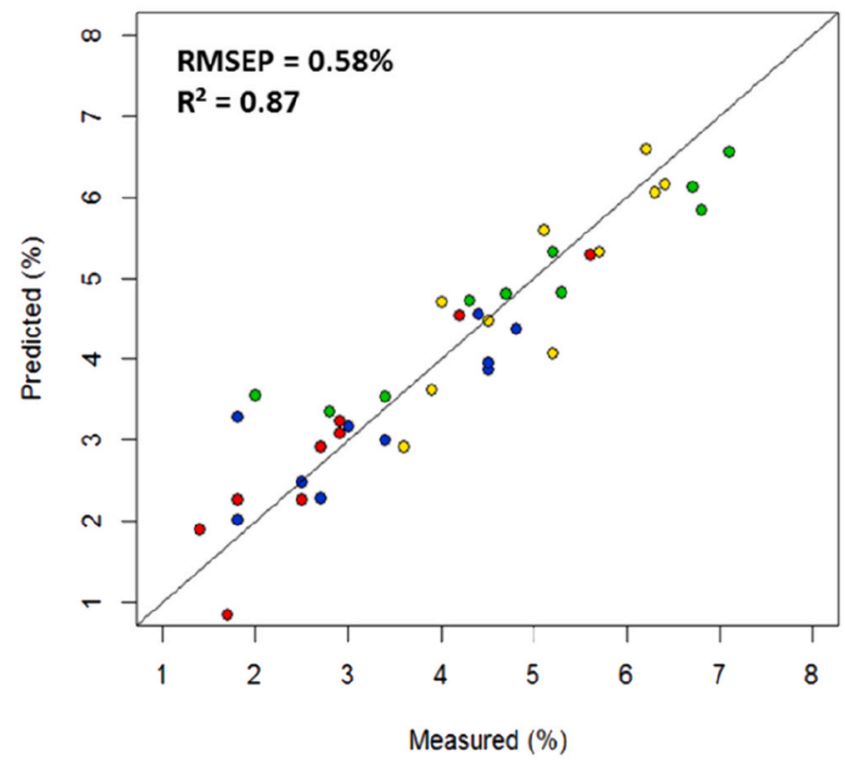

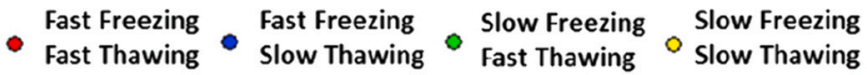

Fig. 6. PLS prediction of liquid loss of data from hyperspectral images taken in the frozen state for a) full spectrum b) wavelength optimized.

A)

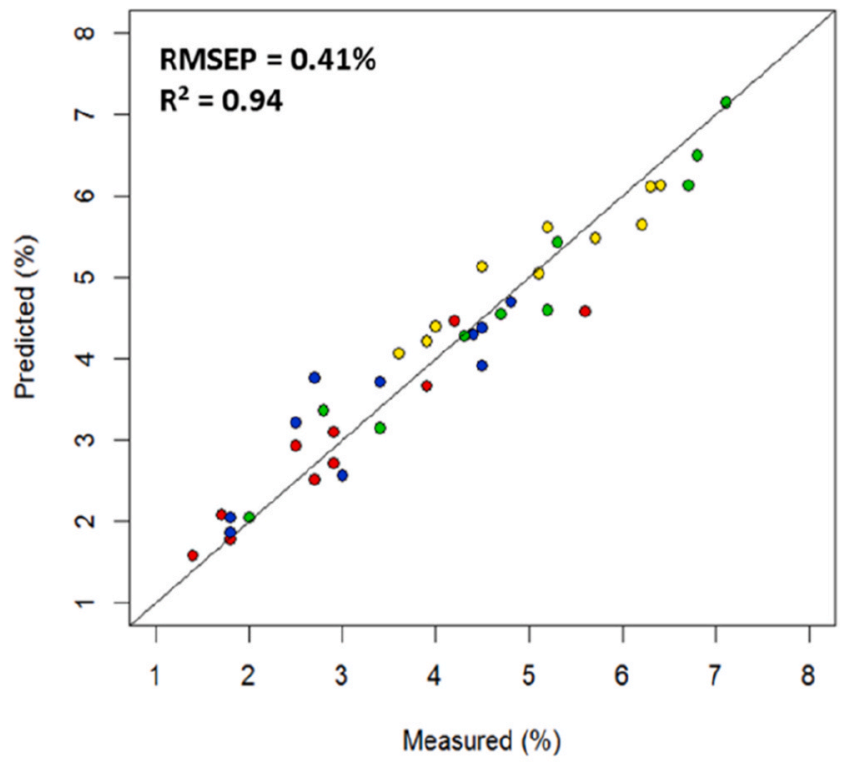

B)

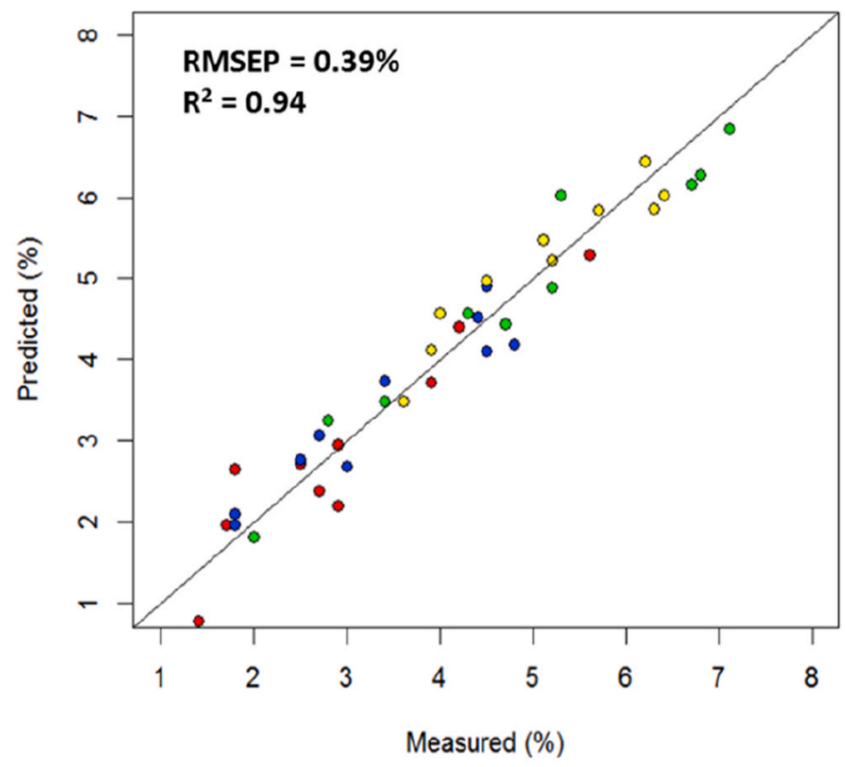

Fast Freezing
Fast Thawing $\begin{aligned} & \text { Fast Freezing } \\ & \text { Slow Thawing }\end{aligned} \bullet \begin{aligned} & \text { Slow Freezing } \\ & \text { Fast Thawing }\end{aligned} \bullet \begin{aligned} & \text { Slow Freezing } \\ & \text { Slow Thawing }\end{aligned}$

Fig. 7. PLS prediction of liquid loss of data from hyperspectral images taken in the thawed state for a) full spectrum b) wavelength optimized.

uncertainty arose in the samples that had one low quality processing (a slow process) and one high quality processing (a fast process). Interestingly, much of the information that allows the samples to be separated by handling protocol was contained in higher components of the principal component analysis, Fig. 10.

\section{Discussion}

Overall, hyperspectral imaging shows a good ability to predict liquid loss in cod that has been frozen and thawed. The $\mathrm{R}^{2}$ and the RMSEP for the PLSR predictions in the fresh and thawed states are similar to results found by other researchers using hyperspectral imaging to predict liquid loss in fish (Cheng et al., 2016; He et al., 2014). The study by He and 
A)

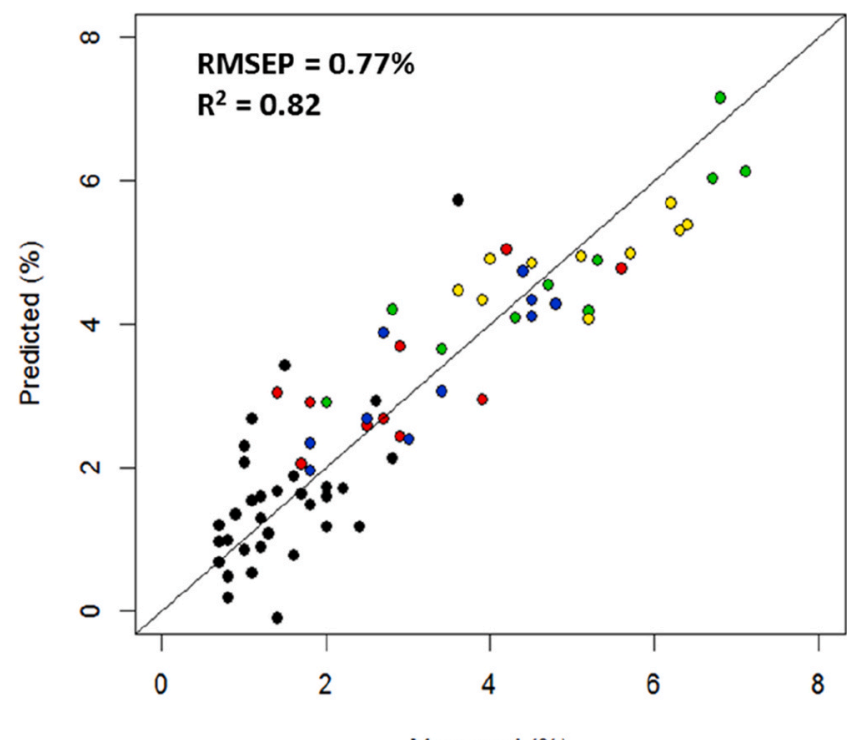

Measured $(\%)$
B)

Wavelength Optimized

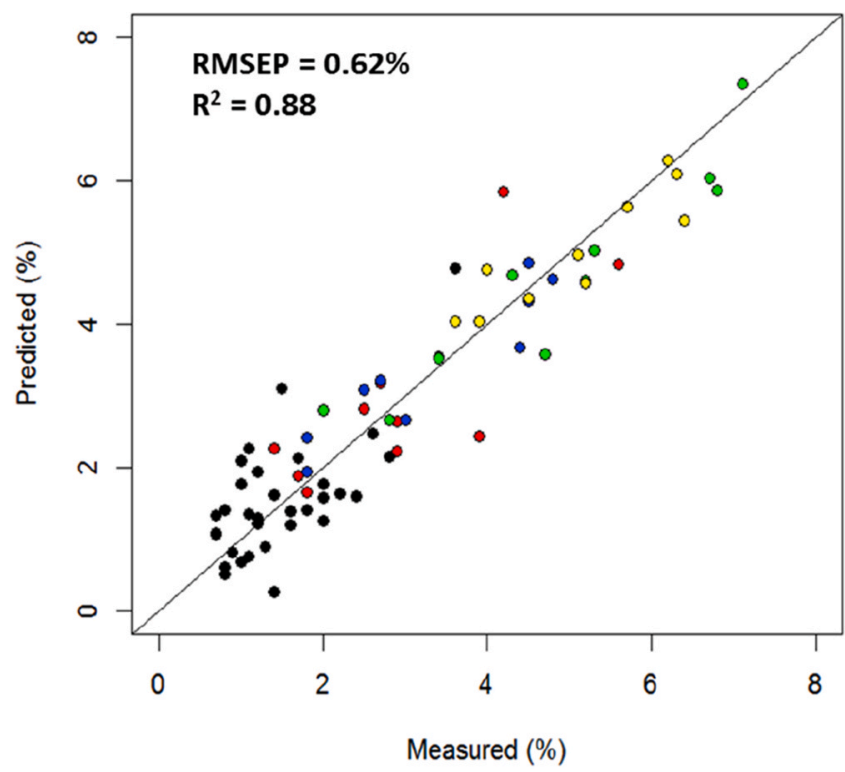

Chilled $\begin{aligned} & \text { Fast Freezing } \\ & \text { Only } \\ & \text { Fast Thawing }\end{aligned}$ Fast Freezing
Slow Thawing Fast Thawing $^{\text {Slow Freezing }} \begin{aligned} & \text { Slow Freezing } \\ & \text { Slow Thawing }\end{aligned}$

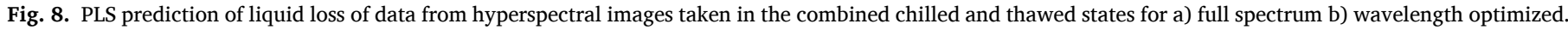

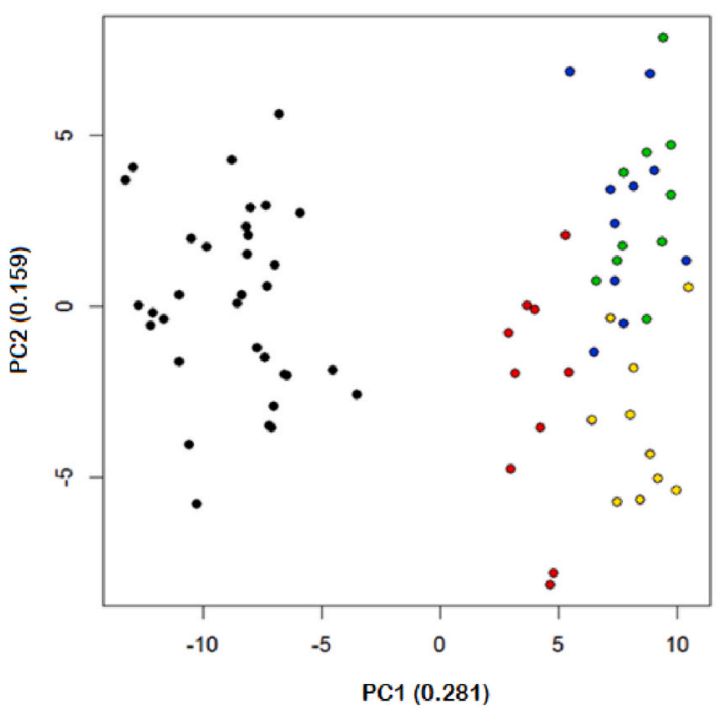

- Chilled Fast Freezing - Fast Freezing - Slow Freezing $0 \begin{aligned} & \text { Slow Freezing } \\ & \text { Only }\end{aligned}$

Fig. 9. Principal component analysis for combined chilled and thawed state spectra.

colleagues also found that a relatively high number of principal components was necessary in order to predict drip loss using the full spectrum and that wavelength optimization help reduce the number of latent factors. The results here show a greater decrease in the number of principal components, suggesting the genetic algorithm is more efficient at selecting relevant wavelengths than the approach of analysing the PLSR regression coefficients. The fluid volumes produced by the different freezing and thawing experiments indicated that the freezing protocol was the predominant factor in the amount liquid loss in the cod fillets. The analysis of the hyperspectral data supported this finding.
There is significant improvement in the ability to predict liquid loss when measuring on the processed fillets after freezing compared to the chilled state. A smaller improvement in prediction is seen when measuring on the processed fillets after thawing compared to the frozen state. This is also in line with what previous research has shown, that the freezing protocol has a larger influence on the liquid loss a sample will experience than the thawing protocol (Zhu, Ramaswamy, Simpson, 2004).

However, despite the impact of freezing and thawing, there is large individual variation in how much liquid loss the fillets experienced. It may be that fillets that are intrinsically less prone to liquid loss also tolerate the freeze thaw cycle better, or there may be two different mechanisms. To try to better understand how the liquid loss relates to structure, the wavelengths used for optimization of the PLS predictions in the different sample states are examined, as shown in Fig. 11.

Due to the high collinearity in the hyperspectral data, minor variation in the exact wavelength chosen typically does not have a significant impact on the resulting model. Instead, the general areas of the spectrum which are selected by optimization tend to be more important. We note that the number of wavelengths selected by the genetic algorithm is higher than found by other researchers that have used wavelength optimization to predict liquid loss (Cheng et al., 2016). However, PLSR was not used in that study, such that its likely the genetic algorithm selects different numbers of wavelengths dependent on the analysis method used. An interesting trend is that the more processing a sample state had experienced, the more wavelengths were used in the optimized model. For the chilled state, only 12 wavelengths were selected, while 17 were selected in the frozen state. For the thawed state, 21 wavelengths were selected by the genetic algorithm, while 28 were required for the combined thawed and chilled model. This suggests a growing complexity of the spectra, and hence the properties of the sample, with regards to liquid loss. For the chilled and frozen state, the majority of wavelengths were selected in the NIR region. For the thawed and combined models, the $400-500 \mathrm{~nm}$ visible region appears to become very important to prediction. This may be due to increased scattering from tissue damage, since scattering effects generally are stronger for short wavelengths. Also, the ability to separate the fillets by both 


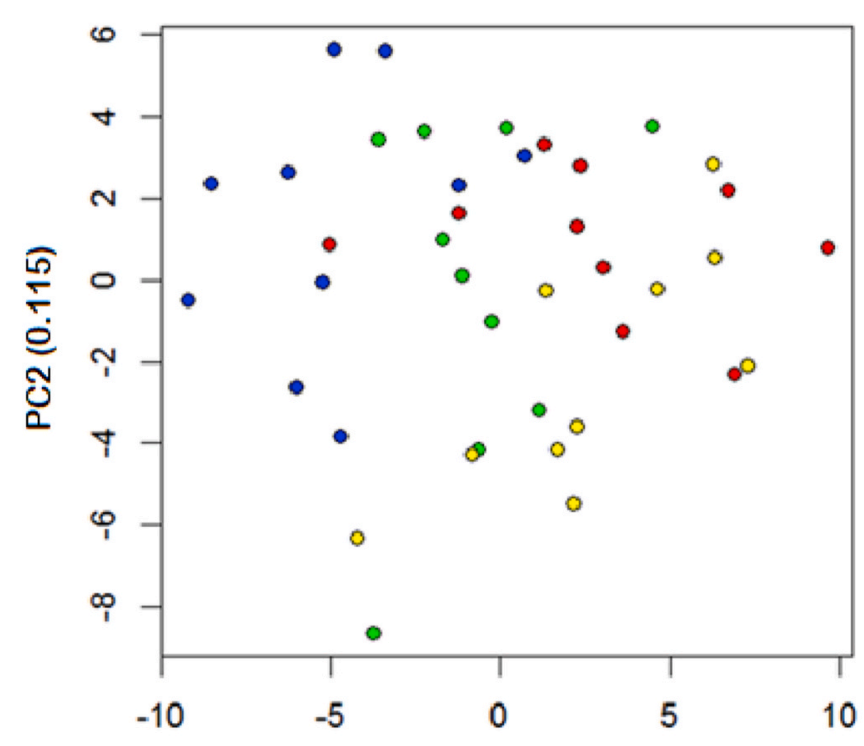

PC1 (0.313)

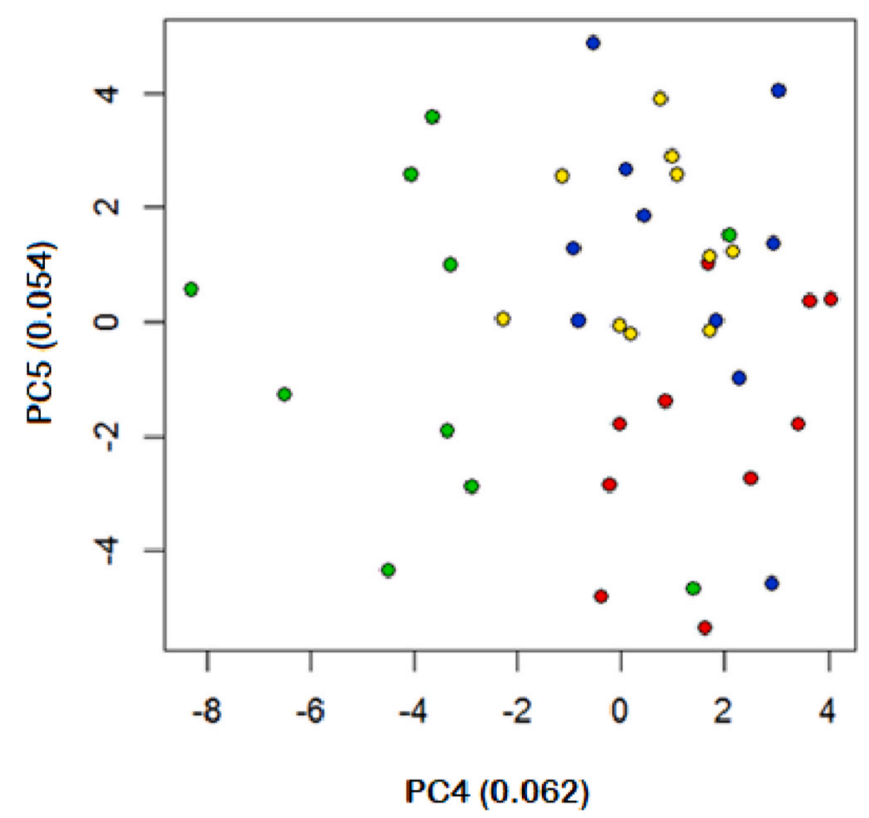

Fast Freezing
Fast Thawing
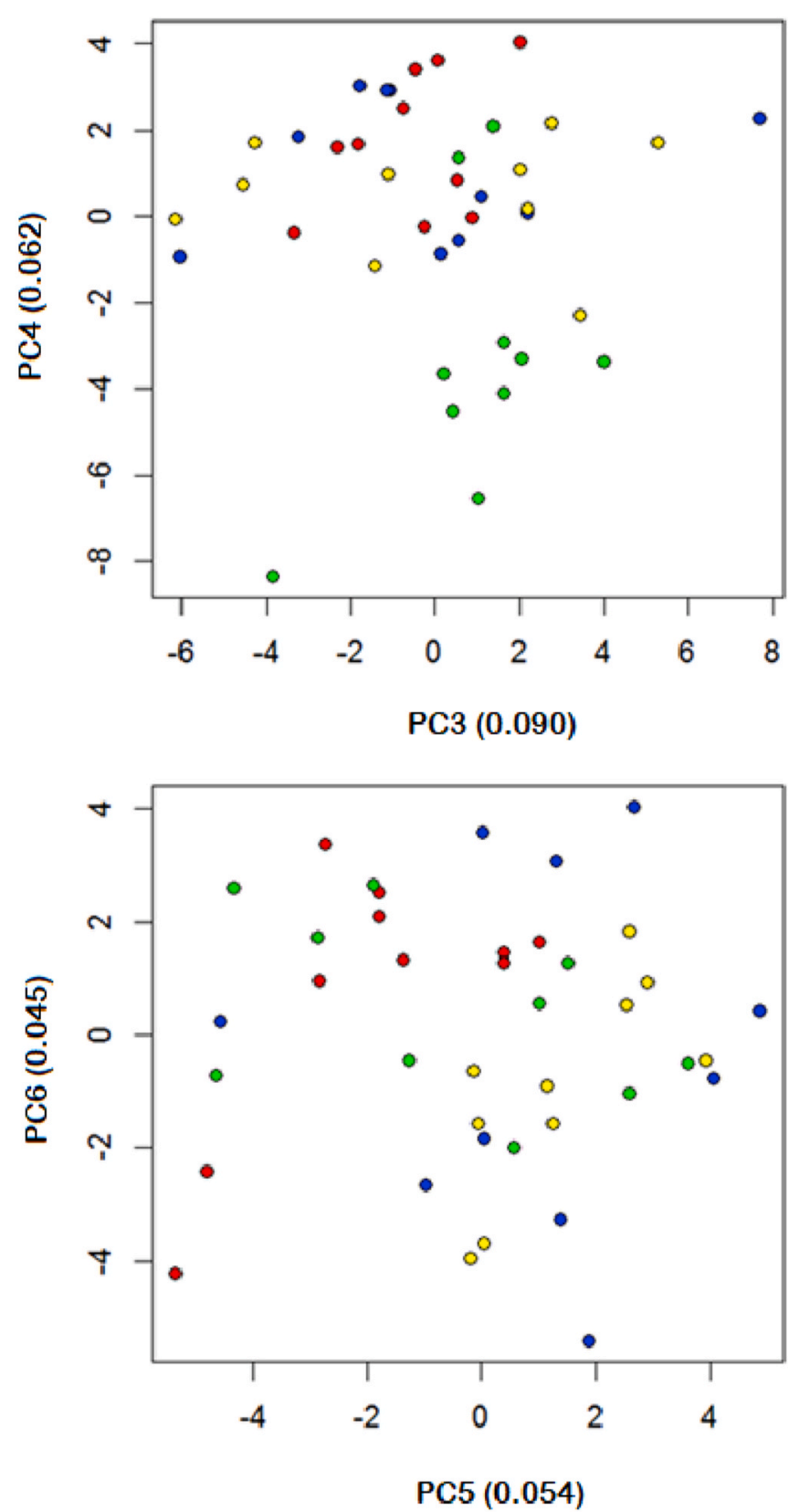

PC5 (0.054)

Fig. 10. PCA analysis of hyperspectral imaging data of thawed samples that have undergone different freezing and thawing protocols.

freezing and thawing protocol in the thawed states indicates that those two processes have different effects upon the tissue. Further study is necessary in order to truly elucidate the relationship between intrinsic sample properties such as chemistry and tissue structure, liquid loss with storage, and response to freezing and thawing.

Reviewing the hyperspectral data indicates that, while it contains information relating to sample liquid loss, this is not the largest source of variance. The full spectrum PLS models required a relatively high number of components in order to produce good predictions. Although sometimes this is simply due to overfitting, the data here indicates it is the result of other sources of variance that must be accounted for by the PLS models, but do not actually contain information relating to liquid loss in the samples. When wavelength optimization was performed, though minor improvements to prediction quality were observed, the biggest improvement was the reduction in necessary components. By removing wavelength sections from analysis, the PLS models were made more efficient, requiring only half the number of components. The PCA plots of the thawed sample data also indicate other sources of variance in the samples, as it was the higher components that contained the information relating to different sample handling. As the study was focused on liquid loss, it is not possible to say what caused the variance, but future studies would benefit in identifying its physical sources, such it can be included in model development and improve the robustness of the multivariate analysis.

While the ability to sort fillets by handling protocol has many benefits, the methodology has possibly even greater benefits if it can be used 


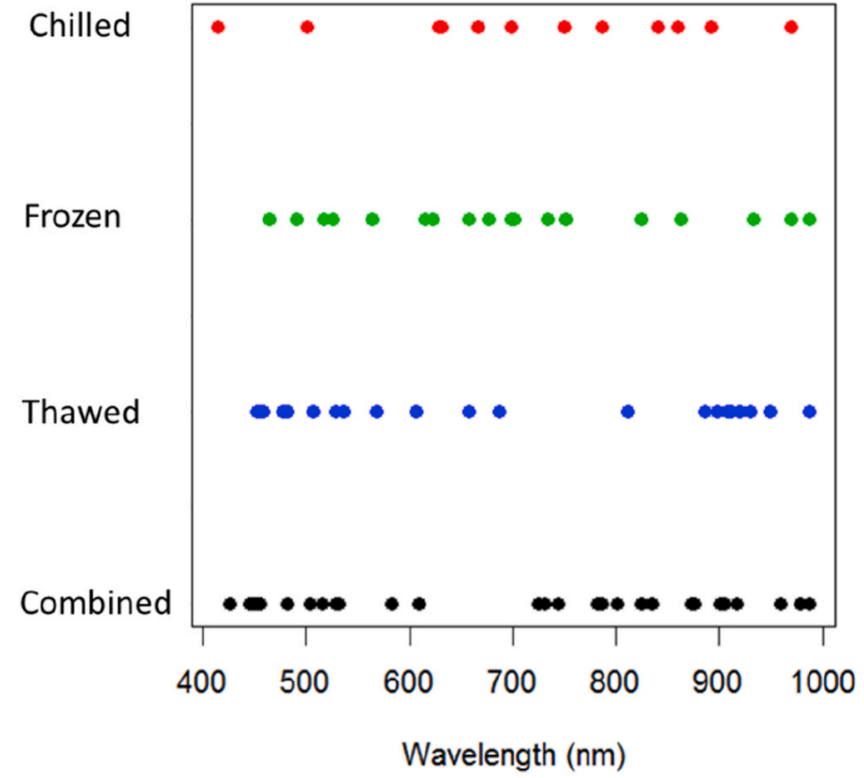

Fig. 11. Wavelengths selected by wavelength optimization for the analyses of the chilled state (Red), frozen state (Green), thawed state (Blue), and combined thawed and chilled states (Black). (For interpretation of the references to colour in this figure legend, the reader is referred to the Web version of this article.)

on whole fish before filleting. Removing fish prone to liquid loss or that have experienced liquid loss before filleting would avoid using production capacity on samples that will later be downgraded. While it is anticipated the methodology should also be applicable to other states than the fillet, future studies are planned to assess the ability of hyperspectral imaging to predict liquid loss on whole or headed and gutted fish. Given the results of $\mathrm{He}$ and colleagues (He et al., 2014), we anticipate that predicting drip loss from hyperspectral imaging on frozen and thawed fish should also be applicable to fatty fish like salmon. Further research is also planned to test the method robustness with regards to typical variations that exist for fish products, e.g. season, catch method, etc.

\section{Conclusions}

Hyperspectral imaging using the interactance mode shows promise as a rapid, non-invasive quality monitoring of vacuum packed cod fillets. This study is the first to demonstrate that the technique can accurately predict liquid loss on unprocessed cod samples in the chilled state and processed cod in the frozen and thawed states - all measured at industrial speeds. Wavelength optimization allowed for modest improvements to prediction and fewer components necessary in developed hyperspectral models. Improvements to the hyperspectral setup enables both freezing and thawing protocol to be determined on thawed samples, enabling producers to better understand the handling history products have experienced. The ability to rapidly sort raw materials has significant implications for the industry. Producers can better set prices for deliveries, sort raw materials into the product line where they will have the most value, and help create final products with consistent quality.

\section{CRediT authorship contribution statement}

Kathryn E. Anderssen: Methodology, Investigation, Formal analysis, Validation, Visualization, Writing - original draft. Svein Kristian Stormo: Conceptualization, Methodology, Investigation, Funding acquisition, Project administration, Resources, Writing - review \& editing. Torstein Skåra: Conceptualization, Methodology, Writing review \& editing. Martin H. Skjelvareid: Software, Resources,
Visualization, Writing - review \& editing. Karsten Heia: Software, Resources, Writing - review \& editing.

\section{Declaration of competing interest}

The authors have no conflicts of interests to declare.

\section{Acknowledgements}

This project is a part of Nofima's Strategic Research Project (SIS) "FRESK", funded by Research Council of Norway (Institute Core Funding).

\section{References}

Altintzoglou, T., \& Heide, M. (2016). Fish quality and consumers: How do consumers' knowledge about and involvement in fish quality define factors that influence fish buying behavior? Journal of Aquatic Food Product Technology, 25, 885-894. https:// doi.org/10.1080/10498850.2014.964432.

Anderssen, E., Dyrstad, K., Westad, F., \& Martens, H. (2006). Reducing over-optimism in variable selection by cross-model validation chem. Intelligent Laboratory Systems, 84 , 69-74. https://doi.org/10.1016/j.chemolab.2006.04.021.

Bangalore, A. S., Shaffer, R. E., \& Small, G. W. (1996). Genetic algorithm-based method for selecting wavelengths and model size for use with partial least-squares regression: Application to near-infrared spectroscopy. Analytical Chemistry, 68, 4200-4212. https://doi.org/10.1021/ac9607121.

Bonilla, A. C., Sveinsdottir, K., \& Martinsdottir, E. (2007). Development of quality index method (QIM) scheme for fresh cod (Gadus morhua) fillets and application in shelf life study. Food Control, 18(4), 352-358. https://doi.org/10.1016/j. foodcont.2005.10.019.

Burger, J., \& Geladi, P. (2007). Hyperspectral NIR image regression part II: Dataset preprocessing diagnostics. Journal of Chemometrics, 20, 106-119. https://doi.org/ 10.1002/cem.986.

Cardello, A. (1995). Food quality: Relativity, context and consumer expectations. Food Quality and Preference, 6, 163-170. https://doi.org/10.1016/0950-3293(94)00039$\mathrm{X}$

Cheng, J. H., Niccolai, B., \& Sun, D.-W. (2017). Hyperspectral imaging with multivariate analysis for technological parameters prediction and classification of muscle foods: A review. Meat Science, 123, 182-191.

Cheng, J. H., \& Sun, D. W. (2014). Hyperspectral imaging as an effective tool for quality analysis and control of fish and other seafoods: Current research and potential applications. Trends in Food Science \& Technology, 37, 78-91. https://doi.org/ 10.1016/j.tifs.2014.03.006.

Cheng, J. H., Sun, D.-W., \& Pu, H. (2016). Combining the genetic algorithm and successive projection algorithm for the selection of feature wavelengths to evaluate exudative characteristics in frozen-thawed fish muscle. Food Chemistry, 19, 855-863.

Cover, T., \& Hart, P. (1967). Nearest neighbor pattern classification. IEEE Transactions on Information Theory, 13, 21-27. https://doi.org/10.1109/TIT.1967.1053964.

Dalvi-Isfahan, M., Jha, P. K., Tavakoli, J., Daraei-Garmakhany, A., Xanthakis, E., \& LeBail, A. (2019). Review on identification, underlying mechanisms and evaluation of freezing damage. Journal of Food Engineering, 255, 50-60. https://doi.org/10.1016/j. jfoodeng.2019.03.011.

Einen, O., Guerin, T., Fjæra, S. O., \& Skjervold, P. O. (2002). Freezing of pre-rigor fillets of Atlantic salmon. Aquacult, 212, 129-140. https://doi.org/10.1016/S0044-8486 (01)00874-2.

ElMasry, G., \& Wold, J. P. (2008). High-speed assessment of fat and water content distribution in fish fillets using online imaging spectroscopy. Journal of Agricultural and Food Chemistry, 56, 7672-7677. https://doi.org/10.1021/jf801074s.

Fagan, J. D., Gormley, R. T., \& Mhuircheartaigh, M. U. (2003). Effect of freeze-chilling, in comparison with fresh, chilling and freezing, on some quality parameters of raw whiting, mackerel and salmon portions. Lebensmittei-Wissenschaft \& Technologie, 36, 647-655. https://doi.org/10.1016/S0023-6438(03)00084-7.

He, H.-J., Wu, D., \& Sun, D. W. (2014). Rapid and non-destructive determination of drip loss and $\mathrm{pH}$ distribution in farmed Atlantic salmon (Salmo salar) fillets using visible and near-infrared (Vis-NIR) hyperspectral imaging. Food Chemistry, 156, 394-401. https://doi.org/10.1016/j.foodchem.2014.01.118.

Hurling, R., \& McArthur, H. (1996). Thawing, refreezing and frozen storage effects on muscle functionality and sensory attributes of frozen cod (Gadus morhua). Journal of Food Science, 61, 1289-1296. https://doi.org/10.1111/j.1365-2621.1996.tb10981.

MacCallum, W. A., Laishley, E. J., Dyer, W. J., \& Idler, D. R. J. P. (1966). Taste panel assesment of cod fillets after single and double freezing. Journal of the Fisheries Research Board of Canada, 23, 1063-1081. https://doi.org/10.1139/f66-097.

Mevik, B. H., \& Wehrens, R. (2007). The PLS package: Principal component and partial least squares regression in R. Journal of Statistical Software, 18, 1-24. https://doi. org $/ 10.18637 /$ jss.v018.i02.

Mevik, B. H., Wehrens, R., \& Liland, K. H. (2019). Pls: Partial least squares and principal component regression. $\mathrm{R}$ package version 2.7-2 https://CRAN.R-project.org/package $=$ pls.

Mørkøre, T., \& Lilleholt, R. (2007). Impact of freezing temperature on quality of farmed atlantic cod (Gadus Morhua L.). Journal of Texture Studies, 38, 457-472. https://doi. org/10.1111/j.1745-4603.2007.00108.x. 
Ofstad, R., Egelandsdal, B., Kidman, S., Myklebust, R., Olsen, R. L., \& Hermansson, A. M. (1996). Liquid loss as effected by post mortem ultrastructural changes in fish muscle: Cod (Gadus morhua L) and salmon (Salmo salar). Journal of the Science of Food and Agriculture, 71(3). https://doi.org/10.1002/(SICI)1097-0010(199607)71:3, 3013123.

Peavy, S., Work, T., \& Riley, J. (1994). Consumer attitudes towards fresh and frozen fish. Journal of Aquatic Food Product Technology, 3, 71-87. https://doi.org/10.1300/ J030v03n02_07.

Petzold, G., \& Aguilera, J. M. (2009). Ice morphology: Fundamentals and technological applications in foods. Food Biophysics, 4(4), 378-396. https://doi.org/10.1007/ s11483-009-9136-5.

Siche, R., Vejarano, R., Aredo, V., Velasquez, L., Saldanam, E., \& Quevedo, R. (2016). Evaluation of food quality and safety with hyperspectral imaging (HSI). Food Engineering Reviews, 8, 306-322. https://doi.org/10.1007/s12393-015-9137-8.

Sivertsen, A. H., Chu, C.-K., Wang, L.-C., Godtliebsen, F., Heia, K., \& Nilsen, H. (2009). Ridge detection with application to automatic fish fillet inspection. Journal of Food Engineering, 90, 317-324. https://doi.org/10.1016/j.jfoodeng.2008.06.035.

Sivertsen, A. H., Kimiya, T., \& Heia, K. (2011). Automatic freshness assessment of cod (Gadus morhua) fillets by Vis/Nir spectroscopy. Journal of Food Engineering, 103, 317-323. https://doi.org/10.1016/j.jfoodeng.2010.10.030.

Skjelvareid, M. H., Heia, K., Olsen, S. H., \& Stormo, S. K. (2017). Detection of blood in fish muscle by constrained spectral unmixing of hyperspectral images. Journal of Food Engineering, 212, 252-261. https://doi.org/10.1016/j.jfoodeng.2017.05.029.

Sun, D. W. (2010). Hyperspectral imaging for food quality analysis and control. Elsevier.

Tsai, B. K., Allen, D. W., Hanssen, L. M., Wilthan, B., \& Zeng, J. (2008). A comparison of optical properties between solid PTFE (Teflon) and (low density) sintered PTFE. In Proc. SPIE 7065, reflection, scattering, and diffraction from surfaces. https://doi.org/ 10.1117/12.798138, 70650Y (August 29, 2008).
Warm, K., Bøknæs, N., \& Nielsen, J. (1998). Development of Quality Index Methods for evaluation of frozen cod (Gadus morhua) and cod fillets. Journal of Aquatic Food Product Technology, 7, 45-59. https://doi.org/10.1300/J030v07n01_04.

Washburn, K. E., Stormo, S. K., Skjelvareid, M. H., \& Heia, K. (2017). Non-invasive assessment of packaged cod freeze-thaw history by hyperspectral imaging. Journal of Food Engineering, 205, 64-73. https://doi.org/10.1016/j.jfoodeng.2017.02.025.

Welch, B. L. (1947). The generalization of "student's" problem when several different population variances are involved. Biometrika, 34(1-2), 28-35. https://doi.org/ 10.1093/biomet/34.1-2.28.

Wold, J. P., Johansen, I. R., Haugholt, K. H., Tschudi, J., Thielemann, J., Segtnan, V. H., et al. (2006). Non-contact transflectance near infrared imaging for representative online sampling of dried salted coalfish (bacalao). Journal of Near Infrared Spectroscopy, 14(1), 59-66.

Xu, J. L., \& Sun, D. W. (2017). Identification of freezer burn on frozen salmon surface using hyperspectral imaging and computer vision combined with machine learning algorithm. International Journal of Refrigeration, 74, 151-164. https://doi.org/ 10.1016/j.ijrefrig.2016.10.014.

Zhu, S., Ramaswamy, H. S., \& Simpson, B. K. (2004). Effect of high-pressure versus conventional thawing on color, drip loss, and texture of Atlantic salmon frozen by different methods. Food Science and Technology, 37, 291-2997. https://doi.org/ 10.1016/j.lwt.2003.09.004.

Zhu, F., Zhang, D., He, Y., Liu, F., \& Sun, D.-W. (2013). Application of visible and near infrared hyperspectral imaging to differentiate between fresh and frozen-thawed fish fillets. Food and Bioprocess Technology, 6, 2931-2937. https://doi.org/10.1007/ s11947-012-0825-6.

Zugarramurdi, A., Parin, M. A., Gadaleta, L., Carrizo, G., \& Lupin, H. M. (2004). The effect of improving raw material quality on product quality and operating costs: A comparative study for lean and fatty fish. Food Control, 15, 503-509. https://doi. org/10.1016/j.foodcont.2003.08.001. 